\title{
A violência familiar sofrida na infância: uma investigação com adolescentes
}

Domestic violence experienced in infancy: an investigation in adolescents

La violencia familiar sufrida en la infancia: una investigación con adolescentes

\author{
Cléa Adas Saliba Garbin* \\ Ana Paula Dossi de Guimarães e Queiroz \\ Tânia Adas Saliba Rovida \\ Orlando Saliba
}

\begin{abstract}
Resumo
Este estudo tem por objetivo evidenciar a prevalência de situações de violência intrafamiliar experimentadas por adolescentes, durante a infância. Trata-se de estudo transversal realizado com 372 adolescentes matriculados em uma instituição de formação profissional de AraçatubaSP. Utilizou-se o Questionário Sobre Traumas na Infância (QUESI) traduzido e validado. Foram investigados ainda gênero e classe social. De acordo com os resultados, 180 adolescentes eram do gênero feminino e 192 do masculino. Predominou a classe social B2 (39,2\%). Setenta e três por cento dos adolescentes relataram ter sofrido pelo menos algum tipo de violência durante a infância. A violência emocional foi a mais prevalente nos graus leve $(28,7 \%)$ e moderado $(9,2 \%)$. Foram encontradas associações entre: violência física e emocional; física e sexual; física e negligência emocional; sexual e emocional; emocional e negligência emocional. Quanto à classe social, não foram encontradas associações. Conclui-se que a maior parte dos adolescentes sofreu abusos na infância, prevalecendo a violência emocional.
\end{abstract}

Palavras-chave: Violência, Violência doméstica, Abuso infantil, Infância, Adolescência.

\begin{abstract}
This study aims to highlight the prevalence of situations of domestic violence experienced by adolescents during their childhood. It is cross-sectional study with 372 adolescents enrolled in a vocational training institution, Araçatuba-SP, utilizing the Childhood Trauma Questionnaire (CTQ), translated and validated. Gender and social
\end{abstract}

* Professores no Departamento de Odontologia Infantil e Social da Faculdade de Odontologia de Araçatuba-SP, professores no Programa de Pós-graduação em Odontologia Preventiva e Social FOA-Unesp. E-mail: cgarbin@foa.unesp.br. 
class were also investigated. According to the results, 180 adolescents were female and 192 were male. The predominant social class was B2 (39.2\%). Seventy two percent of teens reported having experienced at least some form of violence during childhood. Mild (28.7\%) and moderate $(9.2 \%)$ emotional abuse were the most prevalently associated, as well as between: physical and emotional violence, physical and sexual, physical and emotional neglect, sexual and emotional abuse, emotional neglect and emotional. Regarding social class, no associations were found. In conclusion, most teens suffered abuse in childhood, with a prevalence of emotional violence.

Keywords: Violence, Domestic violence, Child abuse, Childhood, Adolescence.

\section{Resumen}

Este estudio tiene como objetivo mostrar la prevalencia de situaciones de violencia intrafamiliares sufrida por adolescentes durante la infancia. Es un estudio transversal con 372 adolescentes matriculados en una institución de formación profesional de Araçatuba-SP. Se utilizó el Childhood Trauma Questionnaire (CTQ), traducido y validado. También se investigó: género y clase social. Según los resultados, 180 adolescentes eran del sexo femenino y 192 del masculino. Predominó la clase social B2 (39,2\%). El 72,3\% de los adolescentes relató haber sufrido por lo menos alguna forma de violencia durante la infancia. La violencia emocional fue la más frecuente en el grado leve $(28,7 \%)$ y moderado (9,2\%). Se encontraron asociaciones entre: violencia física y emocional; física y sexual, física y negligencia emocional; sexual y emocional; emocional y negligencia emocional. En cuanto a clase social, no se encontraron asociaciones. Se concluye que la mayoría de los adolescentes sufrió abusos en la infancia, prevaleciendo la violencia emocional.

Palabras claves: Violência, Violencia en la familia, Abuso de menores, Infancia, Adolescencia.

\section{Introdução}

A partir da década de 1970, a violência passou a ser uma das principais causas de morbi-mortalidade nas grandes cidades brasileiras (Minayo \& Souza, 1998). Em 2005, entre as mortes não naturais, as agressões constituíram-se a primeira causa de óbito na faixa etária de 0 a 19 anos de idade em todo o País (Martins \& Jorge, 2009). Apesar dessa constatação, a 
prevalência oculta da violência familiar ainda distorce significativamente as estatísticas oficiais e a realidade é, por vezes, atenuada.

Mesmo que se tenham certas restriçóes quanto ao conhecimento exato da dimensão da violência familiar contra crianças e adolescentes, existem evidências que reforçam a necessidade de um enfrentamento contundente e imediato do problema. Isso porque, independentemente da forma de abuso físico, psicológico, sexual ou negligência, inúmeras são as consequências dos maus-tratos na infância. Essas afetam significativamente o desenvolvimento das esferas física, social, comportamental, emocional e cognitiva desses sujeitos: futuros adultos (Holt, Buckleyb e Whelana, 2008).

Algumas investigações sugerem que a intensidade dos efeitos depende da conjunção de vários fatores, tais como o desenvolvimento psicológico e a capacidade intelectual da criança; o vínculo afetivo entre o agressor e a vítima; a representação do abuso para a criança e a duração dele; a natureza da agressão ou, ainda, as medidas em curso para a prevenção de agressões futuras (Jespersen, Lalumièrea \& Setob, 2009; Reichenheim, Hasselmann \& Moraes, 1999).

Em meio às medidas para prevenção, destacam-se (a) a elaboração de políticas públicas que previnam a ocorrência dos maus-tratos e (b) o diagnóstico das situações críticas, de modo a intervir nos casos de violência familiar já estabelecida. Ambas as estratégias devem se embasar em dados que dimensionem o problema e apresentem suas principais características.

Sabe-se que o diagnóstico dos maus-tratos na infância é difícil; as crianças tendem a esconder a real causa das lesões, quer por medo, quer por afeto, uma vez que os agressores geralmente são os pais ou responsáveis. Justamente pela dificuldade é que os profissionais que lidam com esse grupo, especialmente os trabalhadores da área da saúde, necessitam estar sempre atentos aos detalhes que podem induzir à confirmação. Talvez outros métodos, que não os de perguntar diretamente, podem se mostrar mais efetivos e, mesmo não confirmando a situação, reforçar e embasar as suspeitas do profissional. Marcas que não correspondem ao relatado pelos responsáveis, acidentes recorrentes e incompatíveis com a idade da criança, vestimentas inadequadas ao clima são fortes indicativos de violência (Ferreira et al., 2001). Além disso, a utilização de instrumentos como o Questionário Sobre Traumas na Infância (QUESI) (Grassi-Oliveira, Stein \& Pezzi, 2006) que viabilizem a identificação dos casos pode ser estimulada entre os profissionais de saúde, dada a facilidade de aplicação e fidelidade dos resultados. Além da finalidade primordial de proteção à criança, a importância de se pesquisarem a prevalência, as causas e 
as consequências dos maus-tratos infantis baseia-se na necessidade de se obter informaçōes sobre essas experiências (Paivio \& Cramer, 2004), justamente para que seja possível a proposição de estratégias que previnam ou minimizem o problema.

Diante dessas constatações, propôs-se a realização deste estudo que teve por objetivo evidenciar a prevalência e as associaçôes de possíveis situaçôes de violência vividas na infância por adolescentes de uma instituição pública de formação profissional de Araçatuba, Estado de São Paulo.

\section{Metodologia}

Trata-se de um estudo transversal, realizado em Araçatuba-SP, Brasil. A amostra consistiu de todos os adolescentes com idade entre 14 a 17 anos, matriculados no ano de 2008 em uma instituição pública de formação profissional do município $(\mathrm{n}=390)$. Nesse local, único no Município, são admitidos jovens a partir dos 14 anos de idade, para treinamento profissional e inserção no mercado de trabalho.

Para coleta dos dados, foi utilizado o Questionário Sobre Traumas na Infância (QUESI), instrumento traduzido e validado (Grassi-Oliveira, Stein \& Pezzi, 2006) com base no original Childhood Trauma Questionnaire (CTQ) (Bernstein et al., 2003). Ele constitui um instrumento autoaplicável para adolescentes (a partir de 12 anos) e adultos no qual o sujeito da pesquisa gradua a frequência de 28 assertivas relacionadas com situações ocorridas na infância em uma escala Likert de cinco pontos. Por meio desse instrumento, é possível a verificação da ocorrência de violência física, sexual, emocional e negligências física e emocional.

A violência intrafamiliar é caracterizada por todo tipo de abuso praticado no contexto da família contra qualquer um dos seus membros. Considera-se violência emocional as agressões verbais às crianças, humilhações, tratamentos degradantes. A violência física se refere à agressão corporal de uma criança por um responsável ou cuidador, causando um risco ou resultando em injúria. $\mathrm{O}$ abuso sexual se refere ao contato sexual em que uma pessoa usa a criança para sua gratificação pessoal. Já a negligência se caracteriza pela falta de cuidados e abandono da criança (Krug, Dahlberg, Mercy, Zwi, \& Lozano, 2003).

O questionário não continha a identificação dos participantes e foi aplicado em ambiente escolar, durante o período de aulas, por um único pesquisador. Foram investigadas também variáveis relativas ao gênero, à idade e à condição socioeconômica. 
Após a coleta dos dados, os questionários que apresentavam alguma deficiência quanto ao preenchimento foram descartados. A amostra final contou com 372 questionários, os quais foram digitados em uma planilha eletrônica criada no software Epi Info, versão 3.5.1. (Dean, Dean, Burton \& Dicker, 1990). Para a apreciação dos dados, foi utilizada a escala proposta pelo instrumento original.

Para a verificação de associações, aplicou-se o teste estatístico qui-quadrado e, nos casos em que o uso deste não se mostrou adequado, o teste Exato de Fisher. A análise estatística foi realizada com auxílio do programa BioEstat, versão 5.0. (Ayres, Ayres Jr., Ayres \& Santos, 2007).

A determinação da classe social foi realizada pelo emprego do Critério Padrão Brasil 2008, proposto pela Associação Brasileira de Empresas de Pesquisa (Associação Brasileira das Empresas de Pesquisa, 2007). Essa classificação leva em conta a posse de bens móveis e anos de estudo do chefe da família. Dessa forma, são descritas cinco categorias (A1, A2, B1, B2, C1, C2, D e E), em ordem decrescente, ou seja, a classe A1 é considerada a mais elevada e a $\mathrm{E}$ a mais desprovida.

Atendendo aos ditames éticos das pesquisas realizadas com seres humanos, o projeto foi submetido ao Comitê de Ética em Pesquisa da Faculdade de Odontologia da Universidade Estadual Paulista, Campus de Araçatuba, e obteve aprovação sob o processo número 2007-01343. O termo de consentimento livre e esclarecido para participação no estudo foi obtido com os responsáveis pelos adolescentes, previamente à aplicação dos questionários.

\section{Resultados}

Entre todos os sujeitos, participaram $180(48,4 \%)$ adolescentes do gênero feminino e 192 (51,6\%) do gênero masculino. A idade deles variou entre $14 \mathrm{e}$ 17 anos (média $=15,5, \mathrm{dp}=1,3$ ).

A classe social predominante foi a denominada B2 (39,2\%, IC 95\%, 34,3$44,4)$, em seguida C1 (24,2\%, IC 95\%, 20,0-28,9), C2 (17,5\%, IC 95\%, $13,8-21,8)$, B1 (11,1\%, IC 95\%, 8,1-14,8), D (4,8\%, IC 95\%, 3,0-7,7) e A2 $(3,2 \%$, IC $95 \%, 1,8-5,7)$.

Do total, 72,3\% (269) relataram sofrer pelo menos um tipo de violência durante a infância, enquanto $27,7 \%$ (103) negaram ter vivido violência de qualquer natureza.

O gráfico 1 representa os tipos de violência distribuídos conforme a intensidade. Nota-se que a violência emocional foi mais prevalente que as demais nos graus leve $(28,7 \%)$ e moderado $(9,2 \%)$. 
Gráfico 1 - Distribuição percentual dos tipos de violência, segundo o grau/intensidade. Araçatuba-SP, 2008.

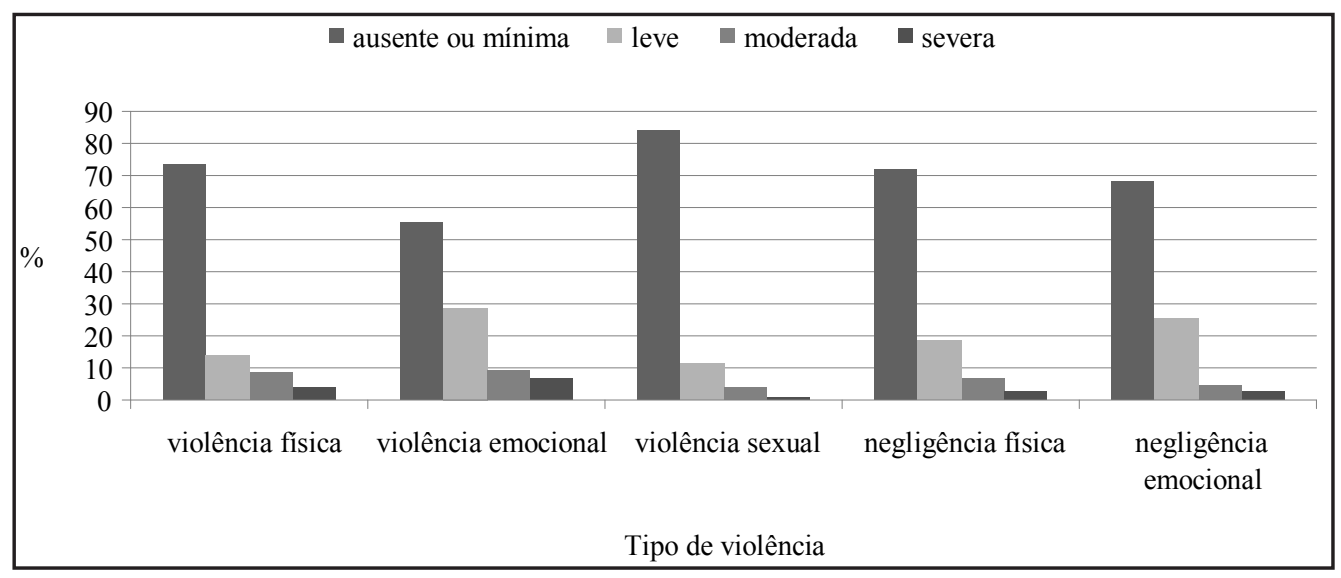

Fonte: Dados da pesquisa

O gráfico 2 evidencia que, durante a infância, os adolescentes sofreram formas combinadas de violência.

Gráfico 2. Distribuição percentual dos adolescentes, segundo os tipos de violência. Araçatuba-SP, 2008.

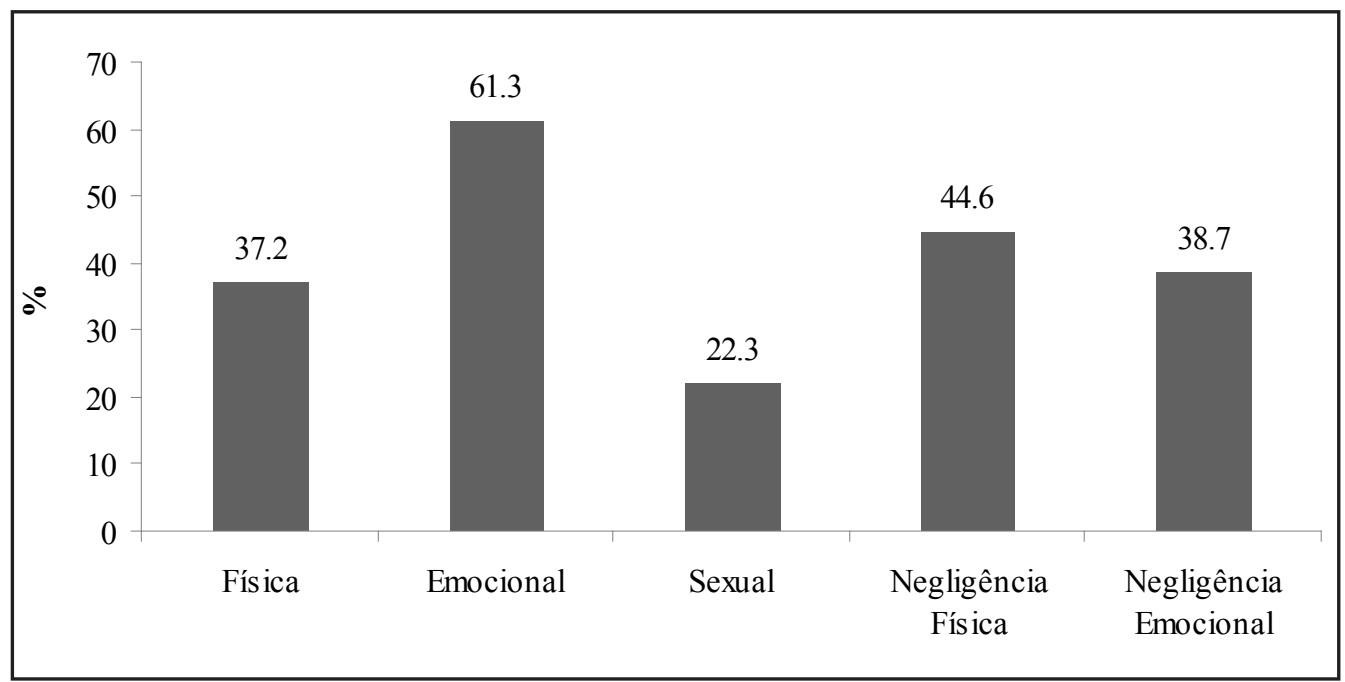

*Poderiam ser apontados um ou mais tipos de violência sofrida

Fonte: Dados da pesquisa

De acordo com a tabela 1, pode-se observar que não foram encontradas associaçôes entre violência e gênero, exceto para a violência emocional e gênero feminino ( $\mathrm{p}=0,0436)$.

Tabela 1 - Distribuição percentual dos tipos de violência segundo gênero. Araçatuba-SP, 2008. 


\begin{tabular}{|c|c|c|c|c|c|c|c|c|c|c|}
\hline \multicolumn{7}{|l|}{ Violência } & \multicolumn{4}{|c|}{ Negligência } \\
\hline Tipo & Física & & Emocion & & Sexual & & Física & & Emoc & onal \\
\hline $\begin{array}{l}\text { Gênero } \\
\text { Grau }\end{array}$ & $\mathrm{F}$ & $\mathrm{M}$ & $\mathrm{F}$ & $M$ & $\mathrm{~F}$ & $M$ & $F$ & $M$ & $F$ & $M$ \\
\hline Ausente & 72,2 & 71,9 & 50,0 & 60,9 & 82,2 & 85,4 & 71,7 & 64,1 & 75,0 & 69,2 \\
\hline Presente & 27,8 & 28,1 & 50,0 & 39,1 & 17,8 & 14,6 & 28,3 & 35,9 & 25,0 & 30,8 \\
\hline$x^{2}$ & 0,1950 & & 4,071 & & Fisher & & 2,1230 & & 1,243 & \\
\hline$p$ & 0,6588 & & $0,0436^{*}$ & & 0,4809 & & 0,1451 & & 0,26 & \\
\hline
\end{tabular}

*Significante

Fonte: Dados da pesquisa

Ao serem analisados os dados da tabela 2, notou-se que foram encontradas associações significativas entre violência física e emocional $(p=0,0001)$; violência física e sexual $(\mathrm{p}=0,0001)$; violência física e negligência emocional $(\mathrm{p}=0,0001)$; violência sexual e emocional $(\mathrm{p}=0,0007)$; violência emocional e negligência emocional $(\mathrm{p}=0,0001)$. Quanto à classe social, não foram encontradas quaisquer associações.

Tabela 2 - Distribuição dos cruzamentos entre os tipos de violência, segundo a significância. Araçatuba-SP, 2008.

\begin{tabular}{|c|c|c|c|}
\hline Cruzamentos* & $x^{2}$ & Probabilidade & Significância** \\
\hline $\begin{array}{l}\text { violência emocional x } \\
\text { violência física }\end{array}$ & 53,751 & 0,0001 & $S$ \\
\hline $\begin{array}{l}\text { violência emocional x } \\
\text { violência sexual }\end{array}$ & 11,377 & 0,0007 & $S$ \\
\hline $\begin{array}{l}\text { violência emocional x } \\
\text { negligência emocional }\end{array}$ & 42,713 & 0,0001 & $S$ \\
\hline $\begin{array}{l}\text { violência emocional x } \\
\text { negligência física }\end{array}$ & 0,001 & 0,9313 & NS \\
\hline $\begin{array}{l}\text { violência física } x \\
\text { violência sexual }\end{array}$ & Fisher & 0,0001 & $S$ \\
\hline $\begin{array}{l}\text { violência física } x \\
\text { negligência emocional }\end{array}$ & 16,510 & 0,0001 & $S$ \\
\hline $\begin{array}{l}\text { violência física x } \\
\text { negligência física }\end{array}$ & 0,4099 & 0,5220 & NS \\
\hline $\begin{array}{l}\text { violência sexual x } \\
\text { negligência emocional }\end{array}$ & Fisher & 0,0507 & NS \\
\hline $\begin{array}{l}\text { violência sexual } x \\
\text { negligência física }\end{array}$ & Fisher & 0,7537 & NS \\
\hline $\begin{array}{l}\text { negligência emocional x } \\
\text { negligência física }\end{array}$ & 1,312 & 0,2520 & NS \\
\hline
\end{tabular}

*Qui-quadrado e Teste Exato de Fisher ao nível de significância de 5\%

**S=significante NS= não significante Fonte: Dados da pesquisa 


\section{Discussão}

Algumas experiências vividas na infância como a frequência de abusos físicos, psicológicos e sexuais podem significar reflexos desastrosos na vida do indivíduo (Anann, 2006; Monteiro, Teles, Castro, Vasconcelos \& Magalhães, 2008). Em determinadas situações, a intervenção oportuna constitui a diferença entre a vida e a morte de uma criança. Infelizmente, a maior parcela das ocorrências violentas se dá entre os laços familiares, o que torna a questão da identificação dos casos ainda mais difícil e relevante, dada a necessidade de se romper os pactos de silêncio impostos nessa situação (Arpini, Soares, Bertê \& Dal Forno, 2008).

Neste estudo pôde-se verificar que a maioria dos adolescentes relatou ser vítima de pelo menos um dos tipos de violência durante a infância. Entre todas as formas, prevaleceu a violência emocional, o que está em concordância com outros estudos semelhantes (Benbenishty, Zeira, Astor \& Khoury-Kassabri, 2002; Tourigny, Hébert, Joly, Cyr, \& Baril, 2008).

Todavia, comparando-se os achados do estudo atual com os números descritos por Gawryszewski e Hidalgo (2008), em pesquisa sobre as notificações compulsórias de violência contra crianças e adolescentes no Estado de São Paulo, nota-se que a violência emocional prevaleceu em apenas $8 \%$ dos casos, ocupando a última colocação. Importante esclarecer que o levantamento das autoras acima levou em conta as formas isoladas, o que não significa que, nas situações de violência física, por exemplo, o abuso emocional não estivesse presente, ainda que não informado.

Outra hipótese para o desacordo é a fonte dos dados. A violência emocional, nesta pesquisa, foi descrita pelas próprias vítimas devido à experiência vivida por elas na infância. A percepção pelas outras pessoas, no entanto, é limitada, já que o abuso emocional não deixa marcas tão evidentes quanto a violência física ou a negligência física.

$\mathrm{Na}$ amostra em questão, a classe social não esteve associada à ocorrência de violência na infância. Questiona-se a ideia de que a violência contra a criança é mais prevalente entre os menos favorecidos economicamente. $\mathrm{O}$ que se sabe é que, nas classes de baixa renda, de uma forma geral, o jovem precisa trabalhar e cuidar de seus irmãos; as famílias são basicamente geridas por mulheres; as meninas têm filhos cedo e, na mesma casa, convivem as três gerações que cuidam e trabalham para o autossustento. Eventos traumáticos ocorrem com frequência: pobreza, muito estresse 
relacionado à sobrevivência, rupturas de vínculos, uso abusivo de drogas, comunidades que convivem com a violência do tráfico de drogas e armas, entre outros (Mascarenhas, Cardoso, Rocha \& Machado, 2006; Cavalcante \& Schenker, 2007).

Quanto à estrutura e aos recursos familiares, pais/mães jovens, solteiros, pobres, desempregados e com nível educacional inferior ao de seus parceiros que não cometem violência têm mais risco de usar a violência contra seus filhos (Avanci, Assis \& Tavares, 2007).

Geralmente crianças menores, do gênero masculino, sofrem mais agressões físicas fatais e não fatais. As meninas correm mais riscos em relação ao infanticídio, ao abuso sexual, à negligência educacional e nutricional, e à prostituição forçada (Avanci, Assis \& Tavares, 2007).

Neste estudo, os adolescentes do gênero masculino sofreram mais violências do que os do gênero feminino, com exceção dos tipos "sexual" e "emocional"; todavia, a diferença quanto ao gênero só foi estatisticamente significante para esta última forma $(\mathrm{p}=0,0436)$. Talvez a característica de sociedade patriarcal com a valorização excessiva dos "filhos homens" em detrimento das meninas possa ter contribuído para que essas adolescentes tenham se sentido rejeitadas.

Aliás, a prevalência de violência sexual entre as meninas está bem acima do encontrado no Brasil, entre 9 e $12 \%$, mas é compatível com o relatado por mulheres peruanas (18-19,5\%), quando perguntadas sobre sofrimento de violência na infância (Pinheiro, 2006). Provavelmente as diversidades socioculturais dos sujeitos e diferenças metodológicas das pesquisas possam explicar essas variações. Além disso, o questionário autoaplicável, como o utilizado neste estudo, pode ter contribuído para a declaração verídica do fato, dado o menor constrangimento causado à vítima.

A literatura tem ratificado que desmembrar as formas de violência sofrida pelas crianças é apenas uma questão conceitual. Na prática, comumente, os vários tipos estão presentes na mesma vítima. Uma criança ou adolescente que é espancado, por exemplo, já sofreu negligência e abuso psicológico; assim como aquela que é abusada sexualmente sofreu também negligência, abuso psicológico e maus-tratos (Silva, 2002).

No mesmo sentido, os achados deste estudo permitem afirmar que a violência física esteve significativamente associada à emocional, à sexual e à negligência emocional. Os casos de abuso sexual relacionaram-se também à violência emocional. Da mesma forma, violência e negligência emocionais. 
Esses achados devem chamar a atenção para dois aspectos: $1^{\circ}$ ) a violência contra a criança ocorre, e os dados a respeito representam apenas uma parcela ínfima dessa realidade; $2^{\circ}$ ) faz-se necessário um aprofundamento da investigação de violências contra crianças, principalmente pelos profissionais que lidam com esses sujeitos. Lesóes físicas ou comportamentos suspeitos podem ser apenas indícios da ocorrência de outras formas de abuso como o sexual, por exemplo. Daí a necessidade de se direcionarem tempo, conhecimento e principalmente empenho na elucidação dos casos.

\section{Conclusão}

Com base nos achados, pode-se concluir que a maioria dos adolescentes pesquisados relatou ter sofrido violência durante a infância. Prevaleceu a violência emocional, nas formas "leve" e "moderada", sendo, entretanto, relatada a combinação dos tipos. Houve associação significativa entre violência física/emocional, física/sexual e física/negligência emocional. Os casos de abuso sexual relacionaram-se também à violência emocional. Da mesma forma, violência e negligência emocionais foram relacionadas.

\section{Referências}

Anann, K. (2006). Preface. In P. S. Pinheiro. World Report on Violence against Children. (pp. 11). Genebra: OMS.

Arpini, D. M., Soares, A. C. O. E., Bertê, L., \& Dal Forno, C. (2008). A revelação e a notificação das situações de violência contra a infância e a adolescência. Psicologia em Revista, 14 (2), 95-112.

Associação Brasileira das Empresas de Pesquisa (2007). Critério padrão de classificação econômica Brasil/2008. São Paulo: ABEP.

Avanci, J. Q., Assis, S. G. \& Tavares, M. (2007). É possível prevenir a violência? Refletindo sobre risco, prevenção e promoção de saúde. In E. R. Souza (ed.). Curso impactos da violência na saúde: bases conceituais e históricas da violência e setor de saúde: Unidade I. (pp. 80-111). Rio de Janeiro: Fiocruz.

Ayres, M., Ayres Jr., M., Ayres, D. L., \& Santos, A. A. S. (2007). BioEstat. Versão 5.0. Aplicaçôes Estatísticas na área das ciências biomédicas. Belém: Sociedade Civil Mamirauá. 
Benbenishty, R., Zeira, A., Astor, R. A. \& Khoury-Kassabri, M. (2002). Maltreatment of primary school students by educational staff in Israel. Child Abuse \& Neglect, 26 (12), 1291-1309.

Bernstein, D. P., Stein, J. A., Newcomb, M. D., Walker, E., Pogge, D. et al. (2003). Development and validation of a brief screening version of the childhood trauma questionnaire. Child Abuse \& Neglect, 27 (2), 169-190.

Cavalcante, F. A. \& Schenker, M. (2007). Violência, família e sociedade. In E. R. Souza (Ed.). Curso impactos da violência na saúde: bases conceituais e históricas da violência e setor de saúde: Unidade I. (pp. 55-77). Rio de Janeiro: Fiocruz.

Dean, A. G., Dean, J. A., Burton, A. H., \& Dicker, R. C. (1990). Epi Info, Version 6: a word processing, database and statistic program for epidemiology on microcomputers, Atlanta: Centers for Disease Control.

Ferreira, A. L., Lopes, Neto, A. A., Silvany, C. M. S., Souza, E. R., Njaine, K., Silva, K.H. et al. (2001). Guia de atuação frente a maus-tratos na infância e na adolescência: orientaçôes para pediatras e demais profissionais que trabalham com crianças e adolescentes. Rio de Janeiro: Sociedade Brasileira de Pediatria.

Gawryszewski, V. P. \& Hidalgo, N. T. R. (2008). Análise das notificaçôes de maus-tratos contra a criança e o adolescente no Estado de São Paulo, 2006. Recuperado em 19 de novembro, 2008, de http://www.webdimensions.com. br/conass/analise_notificacoes_maus-tratos_crianca_adolescente_2006.pdf.

Grassi-Oliveira, R., Stein, L. M., \& Pezzi, J. C. (2006). Tradução e validação de conteúdo da versão em português do Childhood Trauma Questionnaire. Revista de Saúde Pública, 40 (2), 249-255.

Holt, S., Buckleyb, H., \& Whelana, S. (2008). The impact of exposure to domestic violence on children and young people: a review of the literature. Child Abuse \& Neglect, 32(8), 797-810.

Jespersen, A. F., Lalumièrea, M. L. \& Setob, M. C. (2009). Sexual abuse history among adult sex offenders and non-sexoffenders: a meta-analysis. Child Abuse \& Neglect, 33 (3), 179-192.

Krug, E. G., Dahlberg, L. L., Mercy, J. A., Zwi, A. B., \& Lozano, R. (2003). Informe mundial sobre la violencia y la salud. (pp. 66). Washington: Organización Panamericana de la Salud. 
Martins, C. B. G. \& Jorge, M. H. M. (2009). A violência contra crianças e adolescentes: características epidemiológicas dos casos notificados aos conselhos tutelares e programas de atendimento em municípios do Sul do Brasil, 2002 e 2006. Epidemiologia e Serviços de Saúde, 18 (4), 315-334.

Mascarenhas, L. B., Cardoso, F. L., Rocha, G., \& Machado, M. N. M. (2006). Violência e medo permeando a exploração sexual de crianças e adolescentes. Psicologia em Revista, 12 (20), 193-213.

Minayo, M. C. S. \& Souza, E. R. (1998). Violência e saúde como um campo interdisciplinar e de ação coletiva. História, Ciências, Saúde Manguinhos, 4 (3), 513-31.

Monteiro, C. F. S., Teles, D. C. B. S., Castro, K. L., Vasconcelos, N. S. V., \& Magalhães, R. L. B. (2008). Violência sexual contra criança no meio intrafamiliar atendidos no SAMVVIS, Teresina, PI. Revista Brasileira de Enfermagem, 61 (4), 459-463.

Paivio, C. S. \& Cramer, K. M. (2004). Factor structure and reliability of the Childhood Trauma Questionnaire in a Canadian undergraduate student sample. Child Abuse \& Neglect, 28(8), 889-904.

Pinheiro, P. S. (2006). World Report on Violence against Children. Genebra: OMS.

Reichenheim, M. E., Hasselmann, M. H., \& Moraes, C. L. (1999). Consequências da violência familiar na saúde da criança e do adolescente: contribuições para a elaboração de propostas de ação. Ciência e Saúde Coletiva, 4 (1), 109-121.

Silva, L. M. P. (2002). Violência doméstica contra as crianças e os adolescentes. Recife: Edupe.

Tourigny, M., Hébert, M., Joly, J., Cyr, M., \& Baril, K. (2008). Prevalence and co-occurrence of violence against children in the Quebec population. Australian and New Zealand Journal of Public Health, 32 (4), 331-335. 